\title{
SUSTITUCIÓN DE CAPITAL Y TRABAJO, COMPARACIONES DE PRODUCTIVIDAD E INSUMOS PRIMARIOS PROYECTADOS *
}

\author{
Gerard K. Boon \\ El Colegio de México
}

\section{INTRODUCCIÓN}

EN ESTE ARTículo se examina la proporción de los factores de algunos sectores clave de la economía de México conforme a normas internacionales. ${ }^{1}$ El objetivo primario de la investigación consiste en analizar la productividad del trabajo y la tasa de salarios. La productividad del trabajo constituye un factor clave en la estructura económica tanto por razones nacionales como internacionales. La productividad del trabajo proporciona una visión sobre la eficiencia de la producción e indirectamente sobre la intensidad del capital, y expresa además la relación con los salarios y el precio de costo. Externamente puede determinar el grado de competitividad internacional.

Por medio de una función producción se intenta predecir la productividad del trabajo y de los salarios y compararlos con los valores observados cuando se refieren al pasado. Los valores futuros son también proyectados por medio de la función y comparados con valores estimados utilizando funciones y procedimientos diferentes.

* Este artículo constituye una versión actualizada y ampliada de un trabajo realizado por el autor para el Departamento de Programación Industrial de Nacional Financiera en 1968, con un aporte de la Organización para la Investigación Científica en las Regiones Tropicales (wotro-zwo), La Haya, Holanda, y forma parte del libro de próxima publicación: Sector and Technology Choice in Economic Development, con especial referencia al caso de México. El autor agradece las estimulantes discusiones con el licenciado Gerardo Bueno, en ese tiempo director del Departamento.

1 El análisis del presente artículo se refiere a los sectores de industria manufacturera, construcción y minería. La elección se hizo tomando en cuenta las siguientes consideraciones: la industria de manufacturas está llamada a jugar un papel clave en el futuro desarrollo de México; la minería es de particular interés debido a la cuantía de recursos minerales del país, y la construcción es importante particularmente por la facilidad con que esta industria absorbe los excedentes de mano de obra no calificada. No se desconoce la importancia de la agricultura en el desarrollo económico de México. Un tratamiento de este sector es dado en la publicación mencionada.

Un análisis complementario de este trabajo aparece en G. K. Boon, "Factor Intensities in Mexico with Special Reference to Manufacturing", en H. C. Bos (Comp.), Towards Balanced International Growth, Amsterdam-Londres, North Holland Publishing Co., 1969. 


\section{Funciones estadísticas}

\section{Funciones}

En este análisis se utilizan diversas funciones estadísticas. La primera función es conocida comúnmente por los economistas como la función de elasticidad de sustitución constante (CES).

Esta función está aún sujeta a debate y posiblemente su comprobación empírica no está aún totalmente concluida, pero lo mismo se puede afirmar para la mayoría de las herramientas analíticas de la economía. La función alternativa, la Cobb-Douglas, es más directa en cuanto que introduce explícitamente el capital; es también más antigua y se utiliza con mayor frecuencia. Sin embargo, se requiere información sobre el acervo de capital, que no es fácil de obtener con la adecuada desagregación a nivel de sectores para años específicos y, en el caso de una muestra a nivel internacional, para un tamaño de muestra suficiente.

La función cEs relaciona la productividad del trabajo y el salario derivando también la elasticidad de sustitución entre trabajo y capital. ${ }^{2}$

Naturalmente, la función se apoya en diversos supuestos. La función usada en este trabajo implica beneficios constantes de escala. Supone además que todos los países de la muestra tienen acceso al mismo "paquete" de conocimiento tecnológico y que para cierto sector los países están en la misma función de producción.

La función representa una situación de completa libre competencia y los precios de los factores reflejan la productividad marginal de los factores de la producción.

La función ces es más general que la Cobb-Douglas la cual supone una elasticidad de sustitución unitaria y por lo tanto el supuesto de coeficientes de insumo constantes (Walras-Leontief-Harrod-Domar) o en otras palabras, cero sustitución. Sustitución cero y unitaria son casos especiales de la función CES; básicamente la función supone un grado variable de sustitución en cada sector, con la hipótesis de que el coeficiente de sustitución es menor que 1 en la industria manufacturera y mayor que 1 en las actividades primarias.

La función tiene las siguientes propiedades:

1) homogeneidad;

2) elasticidad de sustitución constante entre capital y trabajo;

3) la posibilidad de diferentes elasticidades para diferentes industrias.

Básicamente la función contiene tres parámetros: el de sustitución, el de distribución y el parámetro de eficiencia. En este análisis solamente se estima el primer parámetro.

La segunda función, que será empíricamente probada, pronostica la tasa de salarios por sector a partir del ingreso per capita promedio del país. El supuesto detrás de esta interrelación es que el ingreso per capita

2 Para un tratamiento más amplio de la función ces, véase K. J. Arrow, H. B. Chenery, B. S. Minhas y R. M. Solow, "Capital-Labour Substitution and Economic Efficiency", The Review of Economics and Statistics, agosto de 1961. 
promedio refleja un cierto nivel de desarrollo económico, que a su vez implica una determinada estructura industrial y sectorial. Esta estructura sectorial opera a un nivel de eficiencia y a una remuneración dada del trabajo, y guarda una relación inherente con el ingreso per capita promedio del país y puede además pronosticarse a partir de la última variable.

La tercera función que se utiliza se basa en consideraciones similares. La cuarta función es idéntica a la tercera aunque se introduce una variable explicativa adicional, la población total. Esta última variable incorpora el tamaño del mercado interno en adición al ingreso per capita como una explicación del nivel de productividad en el sector. ${ }^{3}$

Las funciones utilizadas en este trabajo son las siguientes:

$$
\begin{aligned}
& \ln V / l=a+b \ln (W / l) \\
& \ln Y / N=a_{1}+b_{1} \ln \left(W_{1} / l_{1}\right) \\
& \ln (V / l)^{\prime}=a_{2}+b_{2} \ln (Y / N) \\
& \ln (V / l)^{\prime \prime}=a_{3}+b_{3} \ln (Y / N)+c \ln N
\end{aligned}
$$

en donde $V=$ valor agregado bruto

$l '=$ número de trabajadores

$W=$ monto total de salarios

$Y=$ ingreso per capita

$N=$ población total

$a_{1}, a_{2}, a_{3}=$ constantes logarítmicas

$b=$ elasticidad de sustitución entre trabajo y capital

$b_{1}, b_{2}, b_{3}=$ elasticidades (con diferente significado)

\section{La encuesta}

Las funciones anteriores son aplicadas a una muestra de 21 países (la composición de la muestra se presenta en el cuadro 1A del apéndice). Para cada país la industria manufacturera es dividida en 7 sectores siguiendo el esquema de agregación de la matriz de insumo-producto de 15 sectores de la economía mexicana.

Para cada sector el valor agregado bruto (en dólares) es dividido por el número de personas ocupadas o contratadas, dependiendo de la información disponible al respecto. El total de salarios pagados, también en dólares, es dividido por el número total de empleados por. sector. Las cifras se refieren al año de 1965.

Estos dos conjuntos de cifras, el valor agregado bruto por trabajador y la tasa de salarios por trabajador, se requieren para la función cEs, la cual será incorporada en el contexto de la función discutida (véanse los cuadros $1 \mathrm{~A}$ y $2 \mathrm{~A}$ del apéndice). Para la función 2 se requiere también el ingreso per capita, definido como el ingreso nacional dividido por el número de habitantes del país. Estas cifras se presentan en el cuadro $3 \mathrm{~A}$ del apéndice.

En adición a los 7 sectores de manufacturas, las funciones 1, 2 y 3

3 Chenery introdujo esta función en su famoso artículo "Patterns of Industrial Growth", American Economic Review, septiembre de 1960. 
son aplicadas también a los sectores minería y construcción. Para minería y explotación de canteros, las cifras se refieren a 1963, con una muestra de 16 países (la muestra y los datos se presentan en el cuadro 5A). Para el sector de la construcción las cifras se refieren a 1965 y la muestra cubre también 16 países (véase el cuadro 4A).

\section{Los resultados}

Los resultados, obtenidos a partir de la función 1 con la muestra de la industria manufacturera parecen razonables (véase el cuadro 6A) para todos los sectores: los coeficientes de correlación varían entre 0.95 y 0.96 con un coeficiente de determinación cuyos límites son 0.85 y 0.92 . Los errores estándar, con un margen de variación entre un límite de 0.23 hasta 0.31 , son también aceptables.

La elasticidad de sustitución, indicada por los coeficientes $b$, en todos los casos es menor que 1 (varía entre 0.758 y 0.929 ).

Los resultados para la función 2 aparecen en el cuadro $7 \mathrm{~A}$. Los coeficientes de correlación correspondientes son comparables y aun ligeramente mejores para esta función. Los coeficientes $a$ son ahora negativos y los $b$ mayores que la unidad.

Los resultados para la función 3 se presentan en el cuadro 8A. E1 ajuste estadístico es menos apropiado comparado con las otras dos funciones presentadas, pero es enteramente satisfactorio.

Por su parte, los resultados de la función 4 (véase el cuadro 9A) y su ajuste estadístico son significativamente mejores comparados con el de la función 3.

Por último, los resultados de la muestra para el sector minería y construcción se presentan en los cuadros $10 \mathrm{~A}$ y $11 \mathrm{~A}$. Con las funciones 1 y 3 para la minería se obtienen también resultados satisfactorios. De la función 2 se deriva un coeficiente de determinación de 0.266 y un error estándar casi dos veces mayor que el de las otras funciones. En el caso de la muestra para la minería y construcción, la función 4 no está suficientemente probada dado que el tamaño de la población se considera de poca importancia como variable explicativa.

Para la construcción las tres funciones muestran un buen ajuste estadístico.

\section{La elasticidad de sustitución}

De los resultados obtenidos de las funciones estadísticas, el coeficiente $b$, en la función 1, tiene sentido económico importante en cuanto que expresa la elasticidad de sustitución entre capital y trabajo.

La elasticidad de sustitución de los sectores considerados aparece a continuación:

Sector

Industria ligera
Elasticidades de sustitución

0.758

0.803

0.875 


\section{Industria pesada}

$\begin{array}{rlr}7 & \text { Industria química, petróleo y sus derivados } & \\ & \text { e industria del caucho } & 0.912 \\ 8 & \text { Productos minerales no metálicos } & 0.929 \\ 9 & \text { Industria de metales básicos } & 0.802 \\ 10 & \text { Industria de productos metálicos básicos } & \\ & \text { y otras industrias manufactureras } & 0.848\end{array}$

De esta información no pueden derivarse conclusiones definitivas sobre la magnitud de las elasticidades de sustitución entre grupos industriales, pero se observa que en la industria ligera todos los coeficientes son menores que la unidad y que en esta última las elasticidades de sustitución son menores que en la industria pesada.

En un estudio anterior, ${ }^{4}$ en el cual además de elasticidades de sustitución para cada sector de la crru (Clasificación Industrial Internacional Uniforme), se proporcionan coeficientes agregados de la industria manufacturera y se obtienen evidencias más contundentes. Este análisis fue hecho mediante una encuesta extendida a 14 países (abarcando tanto países desarrollados como de escaso desarrollo) con cifras de 1958 sobre elasticidades de sustitución entre trabajo y capital, con los resultados que se indican en el cuadro 1.

\section{Cuadro 1}

MÉxico: Elasticidades de sustitución En la industria ManUfacturera

\begin{tabular}{|c|c|c|}
\hline Clasificación & Grupo & $\begin{array}{c}\text { Mlasticidad } \\
\text { de } \\
\text { Bustitucion }\end{array}$ \\
\hline 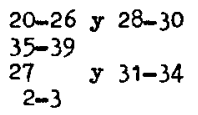 & $\begin{array}{l}\text { Industria ligera } \\
\text { Industria de productos metalicos } \\
\text { Industria pesada } \\
\text { Total manufacturas }\end{array}$ & $\begin{array}{l}0.7211 \\
0.8004 \\
0.8163 \\
0.7427\end{array}$ \\
\hline
\end{tabular}

La información del cuadro 1 muestra un claro incremento en la magnitud de la elasticidad de sustitución tanto en la industria ligera como en la pesada. La industria ligera tiene un coeficiente más bajo que el de la industria manufacturera, y la industria metálica y la pesada tienen un coeficiente mayor que el de las manufactureras en conjunto.

El hallazgo de estas magnitudes, de los coeficientes de sustitución, es interesante ya que se podría pensar en la tendencia contraria: la industria pesada con un coeficiente de sustitución más bajo y la industria ligera con el más alto. Es difícil explicar estas diferencias, pero una razón de que la industria pesada sea más sensible a cambios en los precios de los factores podría ser la posibilidad que existe en este tipo de industrias, con elasticidades de sustitución elevadas, de aumentar la intensidad del trabajo, por ejemplo, implantando turnos múltiples de trabajo.

4 Véase G. K. Boon, "The Place of Small-Scale Industry in the Industrial Framework: A Statistical Analysis", del cual aparece una síntesis en Industrialization and Productivity, Boletín de las Naciones Unidas, Núm. 14, 1969, pp. 66-71. 
Sin embargo, el trabajo calificado en la industria pesada, aun en los países de bajo nivel de desarrollo, podría ser relativamente caro y tener una oferta inelástica, lo que podría inducir a la sustitución de trabajo por capital.

Para la minería, la elasticidad de sustitución es mayor que 1 (1.145) y para la construcción es casi igual a la unidad (0.973). El hecho de que la elasticidad de sustitución para minería sea mayor que la unidad constituye también un hallazgo interesante.

Como se mencionó antes, la hipótesis que se maneja en la función CES, es que la elasticidad de sustitución difiere de sector a sector y que para los sectores de la industria manufacturera es menor que uno (para la producción primaria se considera que es mayor que 1). El análisis presente confirma esta hipótesis.

\section{Comparaciones de PRODUCTIVIDAD}

\section{Las comparaciones para 1965}

a) Industria manufacturera. Utilizando la función ces se puede incorporar la productividad del trabajo observada y pronosticar la correspondiente tasa de salarios y, a partir de ahí, comparar las tasas de salarios observadas con las estimadas y explicar las diferencias.

De manera similar se puede incorporar la tasa de salarios observada y hacer pronósticos sobre la productividad del trabajo para luego comparar los valores de la predicción con los valores observados. Este ejercicio se efectuó hasta 1965, con los resultados que se resumen al final del trabajo.

Para el sector 4 (alimentos elaborados, bebidas y tabaco) la productividad del trabajo observada y la tasa de salarios son de Dls. 1740 y 540, respectivamente, y los valores de la predicción son de Dls. 2020 y 440 . La función internacional sugiere que la productividad del trabajo debe ser más alta o que la tasa de salarios debe ser más baja. Por la manera en que está reglamentada, la tasa de salarios es "inviolable" y por razones políticas muy difícil de reducir. La conclusión es que en 1965, de acuerdo a normas internacionales, la productividad del trabajo era deficiente en el sector 4 y por lo tanto sería preferible aumentarla manteniendo fija la tasa de salarios.

Sin embargo, esta conclusión es preliminar; para confirmarla es necesario investigar qué resultados se obtienen de las funciones 2 y 3 . Por lo que se refiere a la función 2 (véase el cuadro $13 \mathrm{~A}$ del apéndice), mediante su aplicación, se estimó que el ingreso per capita en 1965 era de Dls. 350. Al utilizar este valor en la función para el sector 4, la tasa de salarios correspondiente es de Dls. 740 , sustancialmente más alta que la observada. Aplicando esta tasa de salarios en la función 1, la correspondiente productividad del trabajo que se obtiene es de Dls. 2600 , también sustancialmente más alta que la observada.

Así, de acuerdo a las estimaciones basadas en la función 2, la conclusión es que, de acuerdo con el ingreso per capita de México, en el sector 4 la tasa de salarios debería ser más alta y la productividad del trabajo correspondiente debería ser también mayor de manera sustancial. 
La explicación podría ser que la función 2, en el caso de México, no es adecuada para hacer predicciones, esencialmente porque en el país existe una economía de carácter dual, lo que implica una distribución del ingreso muy desigual - de tal modo que el patrón promedio del ingreso per capita no es un indicador aceptable sobre el desarrollo real del país. Si eliminamos el grupo de ingresos superior, $4 \%$ de la población que en 1963 recibió $23.2 \%$ del ingreso total, el ingreso per capita se reduce a Dls. 300 en 1965. Utilizando este ingreso per capita corregido en la función 2, la tasa de salarios estimada sería de Dls. 650 y utilizando ésta en la función 1, la productividad correspondiente de trabajo es Dls. 2350 , también sustancialmente más alta que la productividad del trabajo observada de Dls. 1740 .

Es de interés observar los resultados que se obtienen al aplicar la función 3 que sirve para pronosticar la productividad del trabajo a partir del ingreso per capita. Los valores de la predicción para el ingreso per capita son Dls. 2750 y 2520 respectivamente.

Antes de analizar los resultados para el sector 4 obsérvese el resumen que aparece en el cuadro 2.

\section{Cuadro 2}

México: Productividad $(v / l)$ y tasa de Salarios $(W / l)$ eN EL SEctor 4 (ALIMENTOS, BEBIDAS Y TABACO), 1965

(Dólares)

\begin{tabular}{|c|c|c|c|c|c|}
\hline \multirow{2}{*}{\multicolumn{2}{|c|}{ Funcion }} & \multicolumn{2}{|c|}{ Valores observados } & \multicolumn{2}{|c|}{ Valores de la prediooión } \\
\hline & & $v / 1$ & $\pi / 1$ & $v / 1$ & $w / 1$ \\
\hline 1 & & 1740 & 530 & 2020 & 440 \\
\hline $\begin{array}{l}2 \\
2\end{array}$ & $\begin{array}{l}Y / \mathbb{N}=350 \\
Y / \mathbb{N}=300\end{array}$ & $\begin{array}{l}1740 \\
1740\end{array}$ & $\begin{array}{l}530 \\
530\end{array}$ & $=$ & $\begin{array}{l}740 \\
650\end{array}$ \\
\hline$\frac{1}{i}$ & $\begin{array}{l}w / 1=740 \\
w / 1=650\end{array}$ & $\begin{array}{ll}1740 \\
1740\end{array}$ & $\begin{array}{l}530 \\
530\end{array}$ & $\begin{array}{ll}2 & 600 \\
2 & 350\end{array}$ & $=$ \\
\hline $\begin{array}{l}3 \\
3\end{array}$ & $\begin{array}{l}\mathrm{Y} / \mathrm{N}=350 \\
\mathrm{Y} / \mathrm{N}=300\end{array}$ & $\begin{array}{ll}1740 \\
1740\end{array}$ & $\begin{array}{l}530 \\
530\end{array}$ & $\begin{array}{l}2750 \\
2750\end{array}$ & - \\
\hline $\begin{array}{l}4 \\
4\end{array}$ & $\begin{array}{l}Y / \mathbf{H}=350 \\
Y / \mathbf{N}=300\end{array}$ & $\begin{array}{l}1740 \\
1740\end{array}$ & $\begin{array}{l}530 \\
530\end{array}$ & $\begin{array}{ll}2 & 680 \\
2 & 460\end{array}$ & - \\
\hline
\end{tabular}

Los resultados que se obtienen mediante las funciones utilizadas indican que la productividad del trabajo en el sector 4 debería ser más alta, es decir, entre Dls. 2000 y 2500 por año. La tasa de salarios apropiada debería ser en este caso entre Dls. 530 y 650.

Para el sector 5, existe sólo una pequeña diferencia entre los valores proyectados utilizando la función 1 y los observados. La productividad del trabajo observada es de Dls. 1340 y la tasa de salarios de 540, pero mientras que a partir de esta tasa de salarios el valor estimado de la productividad es de Dls. 1370 , de la productividad del trabajo observada se deriva una tasa de salarios de Dls. 530. En otras palabras, dada la tasa de salarios observada, la productividad del trabajo debería ser un poco mayor o bien, dado el valor observado de la productividad, la tasa de salarios parece ligeramente elevada.

Sin embargo, la función 2 indica que dado el ingreso per capita promedio que existe en México, la tasa de salarios debería ser sustancialmen- 
te mayor. Aun para el ingreso per capita corregido, por la desigual distribución, la tasa de salarios estimada es sustancialmente mayor que la observada. Siguiendo las mismas etapas del análisis, como en el caso del sector 4, la conclusión es que una productividad del trabajo de Dls. 1600 y la tasa de salarios correspondiente de alrededor de Dls. 630, estaría más en concordancia con el nivel de desarrollo de México que los valores observados.

De acuerdo con la función 1, los valores de la predicción para el sector 6 , indican que a la productividad del trabajo observada corresponde una tasa de salarios de Dls. 860, en tanto que la observada es de Dls. 663. En consecuencia, se puede afirmar que la retribución del trabajo es menor de lo que la productividad del mismo sugiere. La misma conclusión se deriva de los resultados provenientes de la función 2 en tanto que de la función 3 se obtiene un valor más cercano al observado. Las estimaciones para el sector 7 (industria química), utilizando la función 1 , son casi idénticas a los valores observados. En otras palabras, de acuerdo con la función CES, en México la industria química opera en concordancia con las normas internacionales en lo que se refiere a las relaciones entre productividad y tasas de salarios. Sin embargo, las funciones 2 y 3 sugieren que tanto la productividad del trabajo como la tasa de salarios deben ser bastante más altas.

Para el sector 8 se encontró, de acuerdo con la función 1, que la productividad del trabajo observada es más alta que el valor que sugiere la tasa de salarios correspondiente: Dls. 690 en lugar de 580 observados. Las funciones 2 y 3 indican de nuevo que para el ingreso per capita que prevalece en México tanto la productividad como la tasa de salarios deberían ser más elevadas.

Para el sector 9 se concluye que la tasa de salarios es demasiado elevada, en relación a la productividad del trabajo existente. En virtud de que la tasa de salarios que se obtiene de la predicción es de Dls. 820 y el valor observado es de Dls. 920, para restablecer el equilibrio, la productividad del trabajo debe aumentar desde un valor observado de D1s. 2500 al año hasta 2760 . De nuevo, las funciones 2 y 3 indican que tanto la productividad como la tasa de salarios deben ser más altos en México.

Finalmente, esta misma conclusión es válida para el sector $10 . \mathrm{Si}$ se considera que la tasa de salarios es justificada, la productividad del trabajo debería subir de Dls. 1250 a 1370 . Las funciones 2 y 3 sugieren de nuevo que la productividad del trabajo así como los salarios deben ser más elevados.

Los resultados para la industria de manufacturas permiten afirmar que, de acuerdo con la función 1, a cuyo análisis se da prioridad, los sectores que se indican en el cuadro 3 muestran una productividad del trabajo deficiente si se considera que las tasas de salarios observadas son difíciles de modificar.

Para las industrias 6 y 8 (véase el cuadro 3), el análisis sugiere que el trabajo no recibe la retribución que le corresponde, dada la elevada productividad observada.

En el sector 7 (industria química) se observa un balance justo: tanto los valores observados como los valores de la predicción son idénticos. Lo 
mismo sucede en el sector 5 en donde los valores de la predicción y los valores observados son muy semejantes.

Como se observó antes con las tasas de salarios pronosticadas por medio de la función 2, y la productividad del trabajo derivada con la función 3 y con un ingreso per capita dado, se obtienen valores sustancialmente mayores que los observados o que los pronosticados mediante la función 1 .

\section{Cuadro 3}

México: Productividad del trabajo $(v / l)$ y tasas de salarios $(W / l), 1965$ (Dólares)

\begin{tabular}{|c|c|c|c|c|c|c|c|}
\hline & \multicolumn{7}{|c|}{ sootor } \\
\hline & 4 & 5 & 6 & 7 & 8 & 9 & 10 \\
\hline $\begin{array}{l}\text { Valores observado } \\
v / 1 \\
W / 1\end{array}$ & $\begin{array}{r}1740 \\
530\end{array}$ & $\begin{array}{r}1340 \\
540\end{array}$ & $\begin{array}{r}2080 \\
660\end{array}$ & $\begin{array}{r}2700 \\
760\end{array}$ & $\begin{array}{r}1810 \\
580\end{array}$ & $\begin{array}{l}2500 \\
920\end{array}$ & $\begin{array}{r}1250 \\
530\end{array}$ \\
\hline $\begin{array}{l}\text { Valorea es timados } \\
\text { la funoi } 6 \mathrm{n} 1 \\
\text { v/1 } \\
w / 1\end{array}$ & $\begin{array}{r}2020 \\
440\end{array}$ & $\begin{array}{r}1370 \\
530\end{array}$ & $\begin{array}{r}1650 \\
860\end{array}$ & $\begin{array}{r}2720 \\
760\end{array}$ & $\begin{array}{r}1550 \\
690\end{array}$ & $\begin{array}{r}2760 \\
820\end{array}$ & $\begin{array}{r}1370 \\
480\end{array}$ \\
\hline $\begin{array}{l}\text { Tasa de Salarios } \\
\text { ostimada oon la } \mathrm{f} \\
\text { oon } \mathrm{Y} / \mathrm{N}=300 \\
\text { con } \mathrm{Y} / \mathrm{N}=350\end{array}$ & $\begin{array}{l}650 \\
740\end{array}$ & $\begin{array}{l}630 \\
710\end{array}$ & $\begin{array}{l}780 \\
890\end{array}$ & $\begin{array}{l}1000 \\
1130\end{array}$ & $\begin{array}{l}800 \\
910\end{array}$ & $\begin{array}{ll}1 & 000 \\
1 & 130\end{array}$ & $\begin{array}{l}820 \\
930\end{array}$ \\
\hline $\begin{array}{l}\text { Produotividad del } \\
\text { estimada con: }\end{array}$ & & & & & & & \\
\hline $\begin{array}{l}\text { a) Ia funoibn i } \\
\text { oon } \mathrm{Y} / \mathrm{N}=300 \\
\text { oon } \mathrm{Y} / \mathrm{N}=350\end{array}$ & $\begin{array}{l}2350 \\
2600\end{array}$ & $\begin{array}{l}2300 \\
2520\end{array}$ & $\begin{array}{l}2710 \\
2980\end{array}$ & $\begin{array}{l}3260 \\
3590\end{array}$ & $\begin{array}{ll}2 & 770 \\
3 & 050\end{array}$ & $\begin{array}{l}3260 \\
3580\end{array}$ & $\begin{array}{l}2810 \\
3100\end{array}$ \\
\hline b) la funcion 3 & & & & & & & \\
\hline $\begin{array}{l}\operatorname{con} Y / \mathrm{N}=300 \\
\operatorname{con} \mathrm{Y} / \mathrm{H}=350\end{array}$ & $\begin{array}{l}2520 \\
2750\end{array}$ & $\begin{array}{l}1600 \\
1750\end{array}$ & $\begin{array}{l}1990 \\
2200\end{array}$ & $\begin{array}{ll}3 & 770 \\
4 & 150\end{array}$ & $\begin{array}{l}2190 \\
2440\end{array}$ & $\begin{array}{ll}3 & 260 \\
3 & 520\end{array}$ & $\begin{array}{l}2050 \\
2250\end{array}$ \\
\hline c) la funcion 4 & & & & & & & \\
\hline $\begin{array}{l}\operatorname{con} \mathrm{Y} / \mathrm{N}=300 \\
\operatorname{con} \mathrm{Y} / \mathrm{N}=350\end{array}$ & $\begin{array}{l}2460 \\
2680\end{array}$ & $\begin{array}{ll}1 & 620 \\
1 & 770\end{array}$ & $\begin{array}{ll}2 & 050 \\
2 & 210\end{array}$ & $\begin{array}{ll}3 & 750 \\
4 & 130\end{array}$ & $\begin{array}{ll}2 & 100 \\
2 & 330\end{array}$ & $\begin{array}{ll}3 & 240 \\
3 & 500\end{array}$ & $\begin{array}{ll}2 & 100 \\
2 & 330\end{array}$ \\
\hline
\end{tabular}

a Para la derivación de los valores observados véase el cuadro 13 A del apéndice. Las cifras para México usadas en la encuesta son diferentes. Para lograr homogeneidad en la información para todos los países de la muestra se utilizó la misma fuente.

b Estimados utilizando la tasa de salarios obtenida mediante la función 2.

Los valores pronosticados mediante las funciones 3 y 4 son muy parecidos. Un aspecto interesante de la función 4, que incluye como variable el tamaño de la población, es que para la mayoría de los sectores se obtienen productividades un poco más reducidas que las obtenidas en las funciones que excluyen a la variable población. Dado que el tamaño de la población representa el tamaño del mercado, con seguridad, la limitada escala del mercado explica la reducida productividad del trabajo. Si no se incluyera a la población total sino solamente a la población que constituye el sector moderno de la economía, la productividad del trabajo pronosticada estaría a una escala aún más reducida. Si se hiciera esto, la productividad pronosticada se aproximaría a la que se obtuvo con la 
función 1, mediante la cual se estimó la productividad a partir de las tasas de salario pronosticadas a través de la función 2 .

En cuanto al tamaño de la población considerado en la función 4 (42 700000 habitantes), no es aventurado suponer que sólo un tercio de la población participa activamente en el sector moderno de la economía. En este sentido el tamaño efectivo del mercado estaría representado por 14200000 personas. Si se estima la productividad del trabajo utilizando la función 4, considerando $N=14200000$, se obtienen los resultados del cuadro 4.

\section{Cuadro 4}

MÉxico: Productividad del trabajo $(v / l)$ estimada mediante LA FUNCIÓN 4, 1965

(Dólares)

\begin{tabular}{|c|c|c|c|}
\hline Seotor & $\frac{\text { Prod }}{Y / \mathbb{N}=300}$ & $\frac{\mathrm{imgda}}{\mathrm{Y} / \mathrm{s}=350}$ & $\begin{array}{l}\text { Produc tividad } \\
\text { observada }\end{array}$ \\
\hline $\begin{array}{r}4 \\
5 \\
6 \\
7 \\
8 \\
9 \\
10\end{array}$ & $\begin{array}{ll}2 & 600 \\
1 & 570 \\
1 & 860 \\
3 & 760 \\
2 & 300 \\
3 & 220 \\
1 & 970\end{array}$ & $\begin{array}{ll}2 & 830 \\
1 & 720 \\
2 & 200 \\
4 & 140 \\
2 & 550 \\
3 & 480 \\
2 & 180\end{array}$ & $\begin{array}{ll}1 & 740 \\
1 & 340 \\
2 & 080 \\
2 & 700 \\
1 & 810 \\
2 & 500 \\
1 & 250\end{array}$ \\
\hline
\end{tabular}

Los valores de la productividad que aparecen en el cuadro 4 no son muy reducidos. Para los sectores 4,7 y 8 , el parámetro para la variable población es negativo (véase el cuadro $9 \mathrm{~A}$ ) 10 que implica que mientras más reducida sea la población, mayor será la productividad pronosticada del trabajo. La explicación podría ser que no debe introducirse solamente una variable para el mercado interno, sino también una para el mercado externo. Posiblemente para los países pequeños de la muestra el mercado externo sea más importante como variable explicativa, respecto de la magnitud de la productividad, que el mercado interno.

b) Construcción, minería y explotación de canteras. Los valores observados para la industria de construcción que se utilizan en el análisis para 1965 son: productividad del trabajo $=$ Dls. 1520 y tasa de salarios $=850$. Para la industria de la construcción, utilizando la función 1 , la tasa de salarios pronosticada de acuerdo con la productividad observada es de Dls. 910 (véanse los cuadros 4A y 10A) y la productividad del trabajo que corresponde a la tasa de salarios observada es de Dls. 1460.

La conclusión que se deriva de acuerdo con la función CES, es que en la industria de la construcción, el trabajo es retribuido por abajo de la productividad en alrededor de $10 \%$.

Con la función 2, resulta una tasa proyectada de salarios de Dls. 945. De nuevo, si se considera este valor en la función 1 se obtiene una productividad del trabajo de Dls. 1580 . Estos valores son más cercanos a los valores observados, pero en la función 2 se aplica el ingreso per capita ajustado de Dls. 300. Al utilizar el ingreso no ajustado, la tasa de salarios proyectada es de Dls. 1080 y la productividad del trabajo correspondiente de D1s. 1800 . Estos valores se desvían sustancialmente de los va- 
lores observados (fenómeno encontrado también en la industria manufacturera). De la función 3, con ingreso per capita de Dls. 300, se deriva una productividad del trabajo (proyectada) de Dls. 930. Para el ingreso no ajustado, la productividad del trabajo pronosticada es de 1070 .

Los valores pronosticados para 1976 y 1988 así como los resultados para 1965 se presentan en el cuadro 5.

\section{Cuadro 5}

MÉXICO: VALORES PRONOSTICADOS PARA LA INDUSTRIA DE CONSTRUCCIÓN, 1965-1980

(Dólares)

\begin{tabular}{|c|c|c|c|c|c|c|c|c|c|}
\hline Año & $Y / N$ & & $\mathrm{v} / \mathrm{I}$ & Punoión & $v / 1$ & Punoi6n & & $w / 2$ & Funoi 6n \\
\hline $\begin{array}{l}1965 \\
1965 \\
1965 \\
1976 \\
1980\end{array}$ & $\begin{array}{c}- \\
300 \\
350 \\
510 \\
590\end{array}$ & $(1516)^{d}$ & $\begin{array}{ll}1 & 460 \\
1 & 580 \\
1 & 800 \\
2 & 550 \\
2 & 900\end{array}$ & $\begin{array}{l}1 \mathrm{~b} / \\
1 \\
1 \\
1 \\
1\end{array}$ & $\begin{array}{ll} & - \\
1 & 930 \\
1 & 070 \\
1 & 550 \\
1 & 770\end{array}$ & $\begin{array}{l}- \\
3 \\
3 \\
3 \\
3\end{array}$ & $(854)^{\frac{8}{7}}$ & $\begin{array}{r}910 \\
950 \\
1080 \\
1550 \\
1760\end{array}$ & $\begin{array}{l}1 \\
2 \\
2 \\
2 \\
2\end{array}$ \\
\hline
\end{tabular}

a Valores observadas en 1965.

b La función 1 es utilizada en conexión con la 2. Para esta última, se utilizó la tasa de salarios proyectada mediante la función 1 para proyectar las productividades del trabajo.

De estas estimaciones se puede concluir que existen diferencias sustanciales en el resultado de las proyecciones según la función que se utilice.

Parece claro que, cualquiera que sea la razón, la función 3 subestima la productividad del trabajo proyectada. ${ }^{5}$ Por esta razón se considera que la proyección para 1976 y 1980 , realizada con la función 2 en conjunción con la función 1 es la única que tiene validez.

Para el sector minería, al igual que para la construcción fueron ajustadas las mismas funciones. Sin embargo, como el ajuste de la función 2 resultó muy pobre, no se utilizó para las proyecciones (véanse los cuadros $5 \mathrm{~A}$ y $10 \mathrm{~A}$ ).

En este sector, la productividad observada fue de Dls. 2310 y la tasa de salarios observada de 890 . De acuerdo con la función 1, a la productividad observada le corresponde una tasa de salarios de D1s. 950, o sea que también en esta industria, al igual que en la industria de la construcción, el trabajo parece estar subremunerado en alrededor de $10 \%$. A la tasa de salarios observada corresponde a su vez una productividad del trabajo de Dls. 2120.

De las estimaciones sobre la productividad del trabajo mediante la función 3 y de las predicciones de la tasa de salarios, utilizando la función 1, se obtuvieron los resultados que aparecen en el cuadro 6 .

\section{Comparaciones para 1976 y proyecciones hasta 1980}

a) Industria manufacturera. Para comparar la productividad en un año futuro es necesario disponer de varios tipos de proyecciones. En nues-

5 El ajuste estadístico de esta función se experimenta posteriormente. 
Cuadro 6

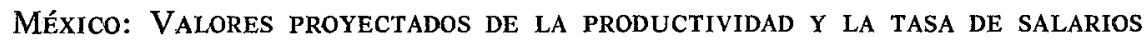
PARA LA INDUSTRIA MINERA Y DE EXPLOTACIÓN DE MINAS Y CANTERAS, 1965-1980

(Dólares)

\begin{tabular}{lccc}
\hline Año & $Y / N$ & $\begin{array}{c}v / 1 \\
\text { (funcion 3) }\end{array}$ & $\begin{array}{c}W / 1 \\
\text { (funcion 1) }\end{array}$ \\
\hline 1965 & 350 & 2710 & 1090 \\
1965 & 300 & 2350 & 960 \\
1976 & 510 & 3950 & 1520 \\
1980 & 590 & 4520 & 1710 \\
\hline
\end{tabular}

tro caso, por medio de las funciones desarrolladas es posible proyectar la tasa de salarios futura si se conoce el ingreso per capita proyectado.

También es posible proyectar la productividad del trabajo directamente con las cifras del ingreso per capita utilizando la función 3 . La tasa de salarios proyectada, obtenida mediante la función 2 , o la productividad derivada de la función 3 pueden utilizarse posteriormente en la función 1 para proyectar tanto la productividad del trabajo como la tasa de salarios.

Una última alternativa disponible, consiste en utilizar una proyección extraoficial de la fuerza de trabajo y del valor agregado para 1976 elaborada por el Banco de México.

A partir de la información sobre fuerza de trabajo, valor agregado y valor agregado por hombre ocupado, se obtienen los resultados del cuadro 7 para la industria manufacturera (véase también el cuadro 12A).

Cuadro 7

MÉXICO: VALOR AGREGADO POR TRABAJADOR EN

LA INDUSTRIA MANUFACTURERA, 1976

(Dólares)

\begin{tabular}{cc}
\hline Sector & $\begin{array}{c}\text { Valor agregado } \\
\text { por trabajador }\end{array}$ \\
\hline 4 & 2030 \\
5 & 2320 \\
6 & 4510 \\
7 & 4890 \\
8 & 3020 \\
9 & 4390 \\
10 & 1520 \\
\hline
\end{tabular}

$\mathrm{Si}$ se supone una tasa de crecimiento del ingreso nacional de alrededor de $7 \%$ y una tasa de crecimiento de la población de $3.5 \%$, el ingreso per capita se incrementará a una tasa de $3.5 \%$. A partir de un ingreso de Dls. 350 en 1965, se obtienen las siguientes proyecciones del producto per capita promedio (en dólares a precios de 1965): 


$\begin{array}{ll}1970 & 420 \\ 1976 & 510 \\ 1980 & 590\end{array}$

Utilizando los últimos dos valores en la función 2, se obtienen las tasas de salarios que aparecen en el cuadro 8.

Cuadro 8

MÉXICo: TASA DE SALARIOS Y PRODUCTIVIDAD DEL tRabajo PRONOSTICADOS, 1976 Y 1980

(Dólares)

\begin{tabular}{|c|c|c|c|c|}
\hline \multirow{2}{*}{ Sector } & \multicolumn{2}{|c|}{1976} & \multicolumn{2}{|c|}{1980} \\
\hline & $\pi / 1$ & $\overline{\nabla / 1}$ & $w / 1$ & $\bar{v} / 1$ \\
\hline $\begin{array}{r}4 \\
5 \\
6 \\
7 \\
8 \\
9 \\
10\end{array}$ & $\begin{array}{r}1050 \\
970 \\
1240 \\
1580 \\
1280 \\
1570 \\
1300\end{array}$ & $\begin{array}{ll}3 & 420 \\
2 & 200 \\
2 & 850 \\
5 & 270 \\
3 & 200 \\
4 & 230 \\
2 & 910\end{array}$ & $\begin{array}{ll}1 & 210 \\
1 & 090 \\
1 & 400 \\
1 & 780 \\
1 & 440 \\
1 & 760 \\
1 & 470\end{array}$ & $\begin{array}{ll}3 & 780 \\
2 & 410 \\
3 & 170 \\
5 & 860 \\
3 & 590 \\
4 & 650 \\
3 & 220\end{array}$ \\
\hline
\end{tabular}

La productividad del trabajo correspondiente puede derivarse de estas tasas de salarios aplicando la función 1 (véase el cuadro 8). Si comparamos estas productividades con las estimaciones derivadas de las proyecciones (completamente diferentes) efectuadas por el Banco de México se observan algunas diferencias de consideración (véase el cuadro 9).

Cuadro 9

MÉXICO: COMPARACIÓN DE PROYECCIONES SOBRE PRODUCTIVIDAD DEL TRABA JO, 1976

(Dólares)

\begin{tabular}{ccc}
\hline Sector & $\begin{array}{c}\text { Proyección estadistica } \\
(\text { funciones 2 } \mathbf{y} \text { 1) }\end{array}$ & $\begin{array}{c}\text { Projecaion del } \\
\text { Banco de Yierico }\end{array}$ \\
\hline 4 & 3420 & 2030 \\
5 & 2200 & 2320 \\
6 & 2850 & 4510 \\
7 & 5270 & 4890 \\
8 & 2210 & 3020 \\
9 & 4230 & 4390 \\
10 & 2910 & 1520 \\
\hline
\end{tabular}

Mientras que en los sectores 5,7 y 8 los resultados son muy semejantes en ambas proyecciones, en los sectores 4,6 y 10 se encuentran diferencias sustanciales.

Por lo que se refiere al sector de productos metálicos, se observa que en 1965 la productividad del trabajo fue de Dls. 1250 mientras que el valor requerido fue de D1s. 1370 . Sin embargo, resulta difícil creer que la productividad del trabajo en los 11 años se hubiera incrementado solamente Dls. 160.

El valor proyectado de la productividad en la industria de productos metálicos en 1976 (Dls. 2900 ) parece razonable. Para la industria de 
productos alimenticios parece demasiado alto, mientras que el valor derivado de la proyección con cifras del Banco es demasiado bajo. La conclusión opuesta se deriva para el sector 6 .

La productividad del trabajo proyectada utilizando directamente la función 3 se indica en el cuadro 10 .

Cuadro 10

México: Productividad del trabajo ( $v / l$ ) proyectada, 1976 y 1980 (Dólares)

\begin{tabular}{lll}
\hline Sector & $1976 \mathrm{a}$ & $1980 \mathrm{~b}$ \\
\hline 4 & 3450 & 3750 \\
5 & 2210 & 2400 \\
6 & 2860 & 3140 \\
7 & 3560 & 5870 \\
8 & 3220 & 3560 \\
9 & 4300 & 4620 \\
10 & 2920 & 3210 \\
\hline
\end{tabular}

a Para $\mathrm{Y} / \mathrm{N}=510$.

b Para $\mathrm{Y} / \mathrm{N}=590$.

Estas proyecciones de la productividad del trabajo son semejantes a las que fueron obtenidas utilizando las funciones 1 y 2 .

b) Construcción, minería y explotación de canteras. Los valores proyectados de la productividad del trabajo en la construcción fueron presentados con anterioridad: la tasa de salarios proyectada utilizada la función 2 (con un ingreso per capita de Dls. 510) fue de Dls. 1550 , y utilizando este valor en la función 1 se obtiene una productividad de Dls. 2550.

La productividad del trabajo estimada con las cifras del Banco de México es de Dls. 2310 , semejante a la obtenida mediante la función 1.

Más aún, debe recordarse que en las cifras del ingreso per capita para 1970 y 1980 no se corrigió la distribución del ingreso como se hizo para 1965. Sin embargo, aunque es cierto que la inequitativa distribución del ingreso continuará en 1970-1980, se espera que sea un poco menos pronunciada que en los años sesenta.

Utilizando cifras corregidas del ingreso per capita en la función 2 podrían resultar tasas de salarios más reducidas $\mathrm{y}$, aplicando éstas en la función 1 deben obtenerse productividades del trabajo reducidas. Sin embargo, aunque no se han agregado estas estimaciones a los numerosos cálculos efectuados en este trabajo, es seguro que la corrección del ingreso per capita, en cuanto a la distribución no equitativa, resultaría en productividades del trabajo 15 o $20 \%$ más bajas, comparadas con los valores presentados que fueron derivados sin estas correcciones.

Las proyecciones para 1980, siguiendo el mismo procedimiento que para 1976, indican una tasa de salarios de DIs. 1760 y una productividad de Dls. 2900.

Los valores pronosticados para 1976 para el sector minería, utilizando las funciones 3 y 1 respectivamente, son: tasa de salarios: Dls. $1520 \mathrm{y}$, 
productividad del trabajo: Dls. 3 950. Con las cifras del Banco de México, la proyección muestra un valor de Dls. 2300 , bastante alejado de las obtenidas.

Los datos proyectados para 1980 son de Dls. 4520 para la productividad del trabajo y Dls. 1710 para la tasa de salarios.

\section{PROYECCIÓN DE INSUMOS PRIMARIOS}

En este trabajo los valores proyectados para la productividad del trabajo que se han obtenido se utilizan ahora para derivar los valores de los insumos del trabajo y se discute un método para derivar, con el material obtenido, el valor de los insumos de capital.

\section{Industria manufacturera}

Para derivar el monto de los insumos se toman como punto de partida los insumos de capital para 1965. Los cuadros 13A y 14A del apéndice muestran cómo se derivan la productividad del trabajo, la tasa de salarios y el cociente capital/trabajo para la industria manufacturera.

El cociente trabajo/valor agregado es justamente el inverso del cociente trabajo/productividad.

El cociente capital/valor agregado se obtiene dividiendo la relación capital/trabajo por la relación trabajo/productividad. En consecuencia:

$$
\frac{k / l}{v / l}=\frac{k}{v}
$$

en donde $k=$ capital, $l=$ trabajo y $v=$ valor agregado.

Del análisis para 1965 hemos aprendido que para los sectores 4, 5, 7,9 y 10 las tasas observadas de salarios fueron algo elevadas en comparación con la productividad del trabajo observada y que lo contrario ' sucede en los sectores 6 y 8.

Por esta razón, en el análisis siguiente se utilizan las tasas de salarios observadas en 1965 para los sectores $4,5,7,9$ y 10, mientras que para los sectores 6 y 8 se utilizan las tasas de salarios pronosticados (véase el cuadro 11).

Por medio de una elaboración posterior de la función CES, se puede pronosticar la relación capital/trabajo para un año futuro, digamos $x$, si se conoce el cociente capital/trabajo, la tasa de salarios en el año base y la tasa de salarios futura en el año $x$.

$\mathrm{Si}$ se supone que la tasa de beneficio del capital permanece igual para el año base y para el año de la proyección $(x)$, la intensidad del capital proyectada puede derivarse mediante la siguiente fórmula: ${ }^{6}$

$$
\ln (k / l)_{x}=b \ln \left(\frac{w_{x}}{w_{o}}\right)+\ln (k / l)_{o}
$$

6 K. J. Arrow, H. B. Chenery, B. S. Minhas y R. M. Solow., op. cit. 
Cuadro 11

MÉxico: TaSa dE SALARIOS EN LA INDUSTRIa MANUfacturera, 1965 (Dólares)

\begin{tabular}{cc}
\hline Sector & Tasa de salarios \\
\hline 4 & 530 \\
5 & 540 \\
6 & 860 \\
7 & 760 \\
8 & 690 \\
9 & 920 \\
10 & 530 \\
\hline
\end{tabular}

La derivación de esta ecuación se obtiene como sigue:

$$
\begin{aligned}
b & =1 /(1+p) \\
w_{o} & =\frac{1-\delta}{\delta}(k / l)_{o}^{p+1} r \\
\frac{w_{x}}{w_{o}} & =\frac{(k / l)_{x}^{p+1}}{(k / l)_{o}^{p+1}} \\
(k / l)_{x}^{p+1} & =\frac{w_{x}}{w_{o}}(k / l)_{o}^{p+1} \\
(k / l)_{x} & =\frac{\left(w_{x}\right)^{1 / 1+p}}{\left(w_{o}\right)}(k / l)_{o} \\
(k / l)_{x} & =\frac{\left(w_{x}\right)^{b}}{\left(w_{o}\right)}(k / l)_{o}
\end{aligned}
$$

en donde:

$$
\begin{array}{ll}
p=\text { parámetro de sustitución } & b=\text { elasticidad de sustitución } \\
\delta=\text { parámetro de distribución } & o=\text { valores observados } \\
r=\text { tasa de beneficio del capital } & x=\text { valores proyectados }
\end{array}
$$

Las cifras para $w_{o}$ ya fueron presentadas; las cifras para: $(k / l) \circ$ y $w_{x}$, en donde $x$ es el año 1976 , son las que aparecen en el cuadro 12 .

Al aplicar la ecuación 5, se obtiene el resultado indicado en la tercera columna del cuadro 12. Si se compara el cociente pronosticado capital/ trabajo para 1976 con los valores observados para 1965, se observa un incremento sustancial. La relación capital/valor agregado se deriva, como se mencionó antes, dividiendo el cociente capital/trabajo por la relación valor agregado/trabajo y la relación trabajo/valor agregado calculando el inverso de la relación valor agregado/trabajo. 
Cuadro 12

México: Relación capital/trabajo proyectada, 1965-1976

(Dólares)

\begin{tabular}{|c|c|c|c|}
\hline Sector & $\begin{array}{c}k / 2 \\
(1965)\end{array}$ & $\begin{array}{c}w_{x} \\
(1976)\end{array}$ & $\begin{array}{l}(k / 1) \times \\
(1976)^{x}\end{array}$ \\
\hline $\begin{array}{r}4 \\
5 \\
6 \\
7 \\
8 \\
9 \\
10\end{array}$ & $\begin{array}{rr}4 & 420 \\
2 & 930 \\
5 & 860 \\
8 & 300 \\
5 & 620 \\
13 & 260 \\
3 & 200\end{array}$ & $\begin{array}{l}1050 \\
1970 \\
1240 \\
1580 \\
1280 \\
1570 \\
1300\end{array}$ & $\begin{array}{rr}7 & 460 \\
4 & 690 \\
8 & 040 \\
16 & 100 \\
9 & 750 \\
20 & 370 \\
6 & 820\end{array}$ \\
\hline
\end{tabular}

Resumiendo algunos de los hallazgos previos y los cocientes trabajo/ valor agregado y capital/valor agregado para 1976 se obtienen las cifras del cuadro 13.

Cuadro 13

MÉXICO: INSUMOS PRIMARIOS OBSERVADOS Y ESTIMADOS PARA LA INDUSTRIA MANUFACTURERA, 1965-1976

\begin{tabular}{|c|c|c|c|c|c|c|c|c|}
\hline \multirow{2}{*}{ Seotor } & \multirow{2}{*}{$\begin{array}{l}1 / v^{8} \\
1965\end{array}$} & \multirow{2}{*}{$\begin{array}{c}v / 2 \\
1965\end{array}$} & \multirow{2}{*}{$\begin{array}{l}1 / v^{2} \\
1976\end{array}$} & \multirow{2}{*}{$\begin{array}{c}v / 2 \\
1976\end{array}$} & \multicolumn{2}{|c|}{$k / 1$} & \multicolumn{2}{|c|}{$k / v$} \\
\hline & & & & & 1965 & 1976 & 1965 & 1976 \\
\hline $\begin{array}{r}4 \\
5 \\
6 \\
7 \\
8 \\
9 \\
10\end{array}$ & $\begin{array}{l}580 \\
750 \\
480 \\
370 \\
550 \\
400 \\
800\end{array}$ & $\begin{array}{ll}1 & 740 \\
1 & 340 \\
2 & 080 \\
2 & 700 \\
1 & 810 \\
2 & 500 \\
1 & 250\end{array}$ & $\begin{array}{l}290 \\
460 \\
350 \\
190 \\
310 \\
240 \\
340\end{array}$ & $\begin{array}{ll}3 & 420 \\
2 & 200 \\
2 & 860 \\
5 & 270 \\
3 & 210 \\
4 & 230 \\
2 & 910\end{array}$ & $\begin{array}{r}4420 \\
2 \quad 930 \\
5860 \\
8300 \\
5620 \\
13260 \\
3200\end{array}$ & $\begin{array}{r}7460 \\
4690 \\
8040 \\
16100 \\
9750 \\
20370 \\
6820\end{array}$ & $\begin{array}{l}2.54 \\
2.19 \\
2.82 \\
3.07 \\
3.11 \\
5.30 \\
2.55\end{array}$ & $\begin{array}{l}2.18 \\
2.14 \\
2.82 \\
3.05 \\
3.04 \\
4.81 \\
2.35\end{array}$ \\
\hline
\end{tabular}

a Trabajadores por millón de dólares de valor agregado. Todas las otras cifras están expresadas en dólares, excepto las últimas dos.

Del análisis de esta información se puede concluir, de acuerdo con el procedimiento delineado en este trabajo, que se requiere un incremento muy considerable de la productividad del trabajo y proporcionalmente también de la relación trabajo/valor agregado. Sin embargo, también es cierto que si en México se puede obtener tal productividad del trabajo, se puede lograr competitividad en los mercados internacionales, dado que los valores pronosticados se derivaron de una encuesta internacional.

Del mismo modo, de acuerdo con nuestro análisis, la creciente productividad del trabajo requiere un incremento sustancial en la relación capital/trabajo. Sin embargo, los requerimientos de capital por unidad de valor agregado, debidos al creciente producto por trabajador deben permanecer aproximadamente iguales a los de 1965.

Las posibles explicaciones de estos hallazgos tienen conexión con diversos problemas claves: el empleo puede inicialmente afectarse de manera adversa; la situación de la balanza de pagos puede mejorarse en la medida en que la productividad del trabajo permitiera a México competir efectivamente en el exterior; los requerimientos de capital aumentarían, pero la inversión extranjera directa podría reducir este problema, lo cual 
sería factible si las exportaciones se incrementan en un corto plazo. $\mathrm{Si}$ las exportaciones aumentan sustancialmente, el empleo puede también incrementarse de modo importante y podría lograrse un nuevo equilibrio a un nivel más elevado de desarrollo económico. Pero un incremento de la productividad de la mano de obra puede obtenerse no sólo aumentando la intensidad del capital. Dada la escasez relativa de capital y la urgencia de aumentar el empleo, otra manera de lograrlo sería utilizar la capacidad instalada hasta su máximo, o mejorando la habilidad técnica de la fuerza de trabajo (véase el párrafo final del artículo).

\section{Construcción y explotación de minas y canteras}

Siguiendo el mismo procedimiento que para la industria manufacturera, pueden observarse los insumos primarios de la construcción y la minería para 1976. La información intermedia aparece en el cuadro $14 \mathrm{~A}$ del apéndice y los resultados obtenidos para los indicadores manejados aparecen en cuadro 14.

\section{Cuadro 14}

MÉXICO: INSUMOS PRIMARIOS ESTIMADOS Y OBSERVADOS EN LA INDUSTRIA DE CONSTRUCCIÓN Y MINERÍA, 1965-1976

\begin{tabular}{lccccccccc}
\hline \multirow{2}{*}{ Seotor } & $1 / v^{3}$ & $v / 1$ & $1 / v^{g}$ & $v / 1$ & \multicolumn{2}{c}{$k / 1$} & & \multicolumn{2}{c}{$k / v$} \\
\hline Mineria & 1965 & 1965 & 1976 & 1976 & 1965 & 1976 & & 1965 & 1976 \\
Construco16n & 430 & 2310 & 250 & 3950 & 4110 & 7020 & & 1.78 & 1.78 \\
\hline
\end{tabular}

a Trabajadores por millón de dólares de valor agregado. Todas las otras cifras están expresadas en dólares, excepto las últimas dos.

El procedimiento aplicado trae como consecuencia un incremento sustancial de la productividad proyectada del trabajo, y en consecuencia una reducción sustancial de la relación trabajo/valor agregado debido al considerable incremento en la intensidad del capital, mientras el coeficiente capital/valor agregado permanece constante. Sin embargo, el pronóstico de la productividad del trabajo se obtiene utilizando cifras sobre el ingreso per capita no corregidas en cuanto a la distribución equitativa del ingreso. Si se hiciera esta corrección, el incremento proyectado en la productividad del trabajo tendría que ser menos pronunciado.

Finalmente, es posible que las productividades proyectadas del trabajo sean poco realistas y no del todo deseables, en la medida en que impliquen una reducción en la capacidad de la industria para generar empleo. De este modo, un aumento en la productividad general, de digamos $20 \%$ durante los 11 años considerados, sería preferible obtenerlo con un incremento menor en la intensidad del capital que a los niveles que se indican en este trabajo. Un aumento de la productividad puede también resultar de una mejor organización interna de las fábricas, o bien de utilizar a plenitud la capacidad instalada y del mejoramiento de la capacidad técnica de la mano de obra mediante la experiencia en el trabajo, así como del mejoramiento de la enseñanza institucional. 


\section{APENDICE}

\section{Cuadro $1 \mathrm{~A}$}

VALOR AGREGADO BRUTO POR TRABAJADOR, * MUESTRA INTERNACIONAL, 1965 (Dólares)

\begin{tabular}{|c|c|c|c|c|c|c|c|}
\hline \multirow{2}{*}{ Paía } & \multicolumn{7}{|c|}{$S \circ 0 t \circ F$} \\
\hline & 4 & 5 & 6 & 7 & 8 & 9 & 10 \\
\hline $\begin{array}{l}\text { Estados Unidos } \\
\text { Sueoia. } \\
\text { Canadá } \\
\text { Australia } \\
\text { Noruega } \\
\text { Finlandia } \\
\text { Reino Unido } \\
\text { Alemania (Rep. Foderal) } \\
\text { Israel } \\
\text { Japón } \\
\text { España } \\
\text { Mexico } \\
\text { Chile } \\
\text { Turquia } \\
\text { Jordania } \\
\text { Colombia } \\
\text { Brasil } \\
\text { Filipinas } \\
\text { Perí } \\
\text { Corea } \\
\text { India }\end{array}$ & $\begin{array}{rr}14 & 793 \\
7 & 289 \\
9 & 889 \\
12 & 621 \\
5 & 431 \\
5 & 490 \\
4 & 683 \\
3 & 490 \\
5 & 367 \\
2 & 941 \\
1 & 457 \\
2 & 043 \\
3 & 744 \\
3 & 752 \\
1 & 367 \\
2 & 859 \\
2 & 091 \\
3 & 073 \\
3 & 327 \\
1 & 878 \\
& 634\end{array}$ & $\begin{array}{ll}7 & 210 \\
3 & 828 \\
5 & 505 \\
7 & 326 \\
3 & 921 \\
3 & 006 \\
2 & 506 \\
7 & 338 \\
3 & 523 \\
1 & 954 \\
1 & 736 \\
2 & 080 \\
1 & 203 \\
2 & 104 \\
1 & 195 \\
& 869 \\
1 & 451 \\
& 970 \\
1 & 832 \\
1 & 167 \\
& 711\end{array}$ & $\begin{array}{rr}11 & 128 \\
5 & 292 \\
8 & 815 \\
11 & 155 \\
4 & 806 \\
4 & 673 \\
3 & 763 \\
8 & 068 \\
4 & 409 \\
3 & 046 \\
1 & 346 \\
1 & 872 \\
3 & 418 \\
2 & 406 \\
1 & 177 \\
1 & 576 \\
1 & 673 \\
1 & 583 \\
2 & 030 \\
1 & 126 \\
\ldots & 715\end{array}$ & $\begin{array}{rr}22 & 262 \\
7 & 404 \\
13 & 709 \\
25 & 555 \\
7 & 706 \\
7 & 665 \\
6 & 166 \\
16 & 674 \\
6 & 564 \\
6 & 371 \\
3 & 242 \\
2 & 938 \\
5 & 543 \\
5 & 721 \\
4 & 199 \\
3 & 883 \\
3 & 895 \\
4 & 992 \\
2 & 910 \\
& 873 \\
1 & 185\end{array}$ & $\begin{array}{rr}13 & 201 \\
5 & 784 \\
11 & 035 \\
12 & 796 \\
6 & 348 \\
4 & 821 \\
3 & 741 \\
7 & 946 \\
6 & 790 \\
3 & 209 \\
1 & 354 \\
2 & 368 \\
3 & 313 \\
2 & 898 \\
2 & 802 \\
1 & 493 \\
1 & 380 \\
3 & 008 \\
2 & 182 \\
& 611 \\
0 & 698\end{array}$ & $\begin{array}{rr}15 & 120 \\
5 & 666 \\
11 & 766 \\
13 & 811 \\
7 & 560 \\
4 & 604 \\
4 & 065 \\
7 & 443 \\
7 & 209 \\
4 & 589 \\
2 & 492 \\
2 & 548 \\
6 & 774 \\
3 & 868 \\
1 & 303 \\
2 & 380 \\
2 & 931 \\
3 & 035 \\
8 & 141 \\
1 & 330 \\
1 & 487\end{array}$ & $\begin{array}{rr}13 & 535 \\
5 & 246 \\
9 & 544 \\
8 & 975 \\
5 & 053 \\
4 & 020 \\
3 & 649 \\
8 & 201 \\
4 & 491 \\
3 & 206 \\
1 & 888 \\
1 & 372 \\
2 & 714 \\
2 & 798 \\
1 & 298 \\
1 & 681 \\
3 & 144 \\
1 & 981 \\
1 & 470 \\
& 822 \\
. & 814\end{array}$ \\
\hline
\end{tabular}

Fuente: Naciones Unidas, The Growth of World Industry, Vol. 1, partes I y II, Nueva York, edición 1967 y Cuadros Nacionales: 1953-1965.

Cuadro $2 \mathrm{~A}$

TASA DE SALARIOS POR TRABAJADOR, MUESTRA INTERNAcIONAL, 1965 (Dólares)

\begin{tabular}{|c|c|c|c|c|c|c|c|c|}
\hline \multirow{2}{*}{ Pafo } & \multirow{2}{*}{ 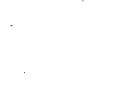 } & \multicolumn{7}{|c|}{ S.otor } \\
\hline & & 4 & 5 & 6 & 7 & 8 & 9 & 10 \\
\hline $\begin{array}{l}\text { Bstados Unidos } \\
\text { Sueoia } \\
\text { Canada } \\
\text { Australia } \\
\text { Noruega } \\
\text { Finlandia } \\
\text { Reino Unido } \\
\text { Alemania (Rep. } \\
\text { Israel } \\
\text { Japon } \\
\text { España } \\
\text { Mexioo } \\
\text { Chilo } \\
\text { Turquia } \\
\text { Jordania } \\
\text { Colombia } \\
\text { Brasil } \\
\text { Filipinas } \\
\text { Pera } \\
\text { Corea } \\
\text { India }\end{array}$ & Foderal) & $\begin{array}{ll}5 & 475 \\
2 & 920 \\
4 & 125 \\
5 & 209 \\
2 & 388 \\
2 & 230 \\
1 & 822 \\
1 & 966 \\
2 & 106 \\
& 879 \\
& 467 \\
& 738 \\
& 749 \\
& 776 \\
& 401 \\
510 \\
\\
& 690 \\
598 \\
884 \\
268 \\
236\end{array}$ & $\begin{array}{ll}3 & 975 \\
2 & 365 \\
3 & 312 \\
4 & 155 \\
2 & 102 \\
1 & 835 \\
1 & 469 \\
1 & 561 \\
1 & 805 \\
& 811 \\
& 716 \\
& 766 \\
& 636 \\
& 799 \\
& 468 \\
& 476 \\
& 512 \\
370 \\
& 724 \\
& 236 \\
442\end{array}$ & $\begin{array}{ll}5 & 759 \\
3 & 039 \\
4 & 692 \\
5 & 798 \\
2 & 452 \\
2 & 473 \\
2 & 220 \\
1 & 952 \\
2 & 310 \\
1 & 215 \\
& 705 \\
& 784 \\
& 954 \\
1 & 137 \\
& 437 \\
& 565 \\
& 795 \\
& 599 \\
& 696 \\
& 387 \\
& 376\end{array}$ & $\begin{array}{ll}6 & 644 \\
3 & 300 \\
5 & 410 \\
8 & 433 \\
2 & 995 \\
2 & 705 \\
2 & 349 \\
2 & 500 \\
2 & 857 \\
1 & 404 \\
1 & 000 \\
1 & 111 \\
1 & 043 \\
1 & 201 \\
& 828 \\
1 & 126 \\
1 & 349 \\
1 & 002 \\
1 & 048 \\
& 315 \\
& 381\end{array}$ & $\begin{array}{ll}5 & 941 \\
3 & 044 \\
4 & 907 \\
5 & 996 \\
2 & 790 \\
2 & 554 \\
2 & 073 \\
2 & 098 \\
2 & 778 \\
1 & 145 \\
& 725 \\
& 899 \\
& 922 \\
863 \\
622 \\
798 \\
610 \\
733 \\
804 \\
313 \\
321\end{array}$ & $\begin{array}{ll}7 . & 360 \\
3 & 480 \\
5 & 607 \\
6 & 460 \\
3 & 059 \\
2 & 734 \\
2 & 315 \\
2 & 321 \\
3 & 023 \\
1 & 722 \\
1 & 036 \\
1 & 079 \\
1 & 773 \\
1 & 112 \\
1 & 477 \\
& 845 \\
& 870 \\
& 691 \\
1 & 292 \\
& 439 \\
& 559\end{array}$ & $\begin{array}{ll}6 & 942 \\
3 & 315 \\
5 & 023 \\
5 & 639 \\
2 & 899 \\
2 & 633 \\
2 & 124 \\
2 & 032 \\
2 & 310 \\
1 & 223 \\
943 \\
700 \\
782 \\
757 \\
7397 \\
851 \\
1080 \\
108 \\
708 \\
736 \\
374 \\
437\end{array}$ \\
\hline
\end{tabular}

Fuente: Tomado de Naciones Unidas, The Growth of World Industry, Vol. 1, partes I y II, Nueva York, edición 1967 y Cuadros Nacionales: 1953-1965 de la misma obra. 
Cuadro $3 \mathrm{~A}$

INGRESO per capita $\mathrm{Y}$ POBLACIÓN ${ }^{\text {a }}$ PARA UNA MUESTRA dE PAÍSES

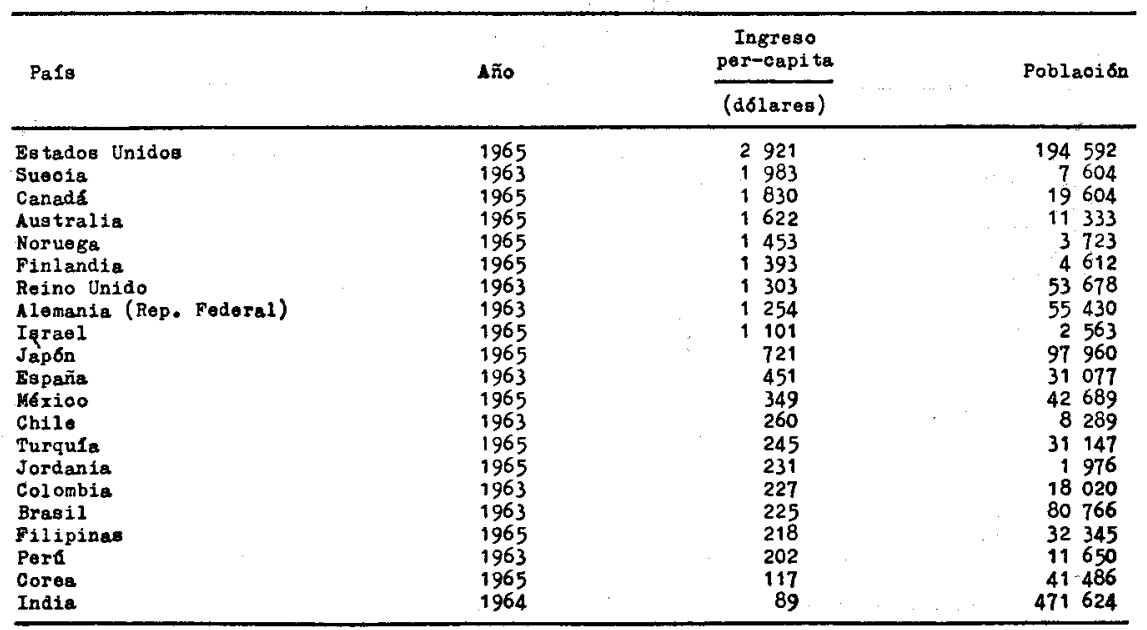

Fuente: Naciones Unidas, Anuario Estadístico, 1967-1969.

a En miles de habitantes.

\section{Cuadro $4 \mathrm{~A}$}

VALOR AGREgado Y TASA DE SALARIOS PARA EL SECTOR DE CONSTRUCCIÓN, 1965

(Dólares)

\begin{tabular}{|c|c|c|c|}
\hline Pais & $\nabla / 2$ & $\pi / 2$ & $\mathrm{Y} / \mathrm{y}$ \\
\hline 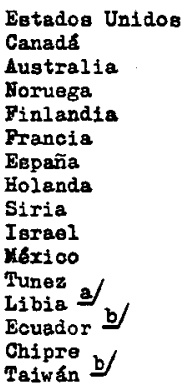 & $\begin{array}{rr}7 & 297 \\
8 & 624 \\
11 & 547 \\
4 & 881 \\
3 & 339 \\
4 & 595 \\
1 & 169 \\
3 & 013 \\
499 \\
2514 \\
1516 \\
1574 \\
1979 \\
1879 \\
1472 \\
938 \\
220\end{array}$ & $\begin{array}{rl}5 & 309 \\
5 & 217 \\
8478 \\
2856 \\
2 \\
2456 \\
1614 \\
1651 \\
1647 \\
4418 \\
1565 \\
& 854 \\
& 577 \\
& 958 \\
& 507 \\
& 845 \\
154 & \end{array}$ & $\begin{array}{l}2921 \\
1830 \\
1622 \\
1453 \\
1393 \\
1528 \\
1589 \\
1280 \\
160 \\
1101 \\
412 \\
177 \\
666 \\
190 \\
600 \\
199\end{array}$ \\
\hline
\end{tabular}

Fuente: Naciones Unidas, Anuario Estadístico, Nueva York, 1967-1969. a 1964.

b 1966 . 


\section{Cuadro $5 \mathrm{~A}$}

\section{VALOR AGREGADO Y TASA DE SALARIOS EN LA MINERÍA Y EXPLOTACIÓN DE MINAS Y CANTERAS, 1963}

(Dólares)

\begin{tabular}{|c|c|c|c|}
\hline Pafa & $v / 1$ & $\pi / 1$ & $y / N$ \\
\hline $\begin{array}{l}\text { Estados Unddos } \\
\text { Canade } \\
\text { Australla } \\
\text { Noruega } \\
\text { Finlandia } \\
\text { Japon } \\
\text { Sud Africa } \\
\text { MExico } \\
\text { Perv } \\
\text { India } \\
\text { Rodesia del Sur } \\
\text { Corea } \\
\text { Paquistán } \\
\text { Iarael } \\
\text { Jordania } \\
\text { Pilipinas }\end{array}$ & $\begin{array}{rl}25 & 079 \\
19 & 804 \\
16 & 171 \\
6 & 086 \\
6 & 192 \\
2 & 342 \\
1 & 704 \\
2 & 654 \\
4 & 194 \\
& 473 \\
1 & 050 \\
1 & 124 \\
& 912 \\
5 & 388 \\
1 & 050 \\
2 & 289\end{array}$ & $\begin{array}{l}6309 \\
4896 \\
6101 \\
2715 \\
2 \quad 257 \\
1008 \\
579 \\
884 \\
1030 \\
105 \\
265 \\
567 \\
451 \\
522 \\
2831 \\
270 \\
770 \\
669\end{array}$ & $\begin{array}{r}2562 \\
1602 \\
1472 \\
1205 \\
1129 \\
576 \\
225 \\
349 \\
202 \\
78 \\
227 \\
129 \\
82 \\
836 \\
189 \\
235\end{array}$ \\
\hline
\end{tabular}

Fuente: Naciones Unidas, Anuario Estadistico, UNO, Nueva York, 1965-1967.

Cuadro $6 \mathrm{~A}$

Resultados DE LA FUnCión 1: $\ln (v / l)=a+b \ln (W / l)$, CON LAS CIFRAS DE LOS PAÍSES DE LA MUESTRA, 1965

\begin{tabular}{|c|c|c|c|c|c|c|c|}
\hline \multirow[t]{2}{*}{. } & \multicolumn{7}{|c|}{$S$ e tote $\mathrm{s}^{\mathrm{g}}$} \\
\hline & 4 & 5 & 6 & 7 & 8 & 9 & 10 \\
\hline \multicolumn{8}{|l|}{$\begin{array}{l}\text { Coefioientes de } \\
\text { regresion }\end{array}$} \\
\hline$\frac{\mathrm{a}}{\mathrm{b}}$ & $\begin{array}{l}2.855 \\
0.758\end{array}$ & $\begin{array}{l}2.169 \\
0.803\end{array}$ & $\begin{array}{r}1.725 \\
0.875\end{array}$ & $\begin{array}{l}1.855 \\
0.712\end{array}$ & $\begin{array}{l}1.427 \\
0.929\end{array}$ & $\begin{array}{l}2.450 \\
0.802\end{array}$ & $\begin{array}{l}1.893 \\
0.848\end{array}$ \\
\hline $\begin{array}{l}\text { Coeficientes } \\
\text { de oorrelacion } \\
\text { de determinaoion }\end{array}$ & $\begin{array}{l}0.935 \\
0.874\end{array}$ & $\begin{array}{l}0.923 \\
0.851\end{array}$ & $\begin{array}{l}0.961 \\
0.924\end{array}$ & $\begin{array}{l}0.932 \\
0.869\end{array}$ & $\begin{array}{l}0.949 \\
0.901\end{array}$ & $\begin{array}{l}0.922 \\
0.851\end{array}$ & $\begin{array}{l}0.943 \\
0.890\end{array}$ \\
\hline Error estándar & 0.278 & 0.285 & 0.225 & 0.306 & 0.281 & 0.281 & 0.271 \\
\hline Prueba $t$ & 11.463 & 10.420 & 15.215 & 11.250 & 13.137 & 10.392 & 12.381 \\
\hline Grados de libertad & 20 & 20 & 20 & 20 & 20 & 20 & 20 \\
\hline
\end{tabular}

${ }^{a}$ Las industrias incluidas en cada sector, de acuerdo con la Clasificación Industrial Internacional Uniforme, son:

Sector 4: Alimentos, bebidas y tabaco (grupos 20, 21 y 22).

Sector 5: Textiles, calzado y vestuario $\mathrm{e}$ industrias del cuero (grupos 23, 24

Sector 6: Madera, muebles, papel, imprentas e industria editorial (grupos 26, 27 y 28).

Sector 7: Productos químicos, petróleo y derivados del petróleo y productos de

Sector 8: Productos minerales no metálicos (grupo 33).

Sector 9: Industrias metálicos básicas (grupo 34).

Sector 10: Productos metálicos, maquinaria, equipo y manufacturas diversas (grupos 35, 36, 37, 38 y 39). 
Cuadro $7 \mathrm{~A}$

Resultados DE LA FUNCión 2: $\ln (Y / N)=a_{1}+b_{1} \ln (W / l)$, CON LAS CIFRAS DE LOS PAÍSES DE LA MUESTRA, 1965

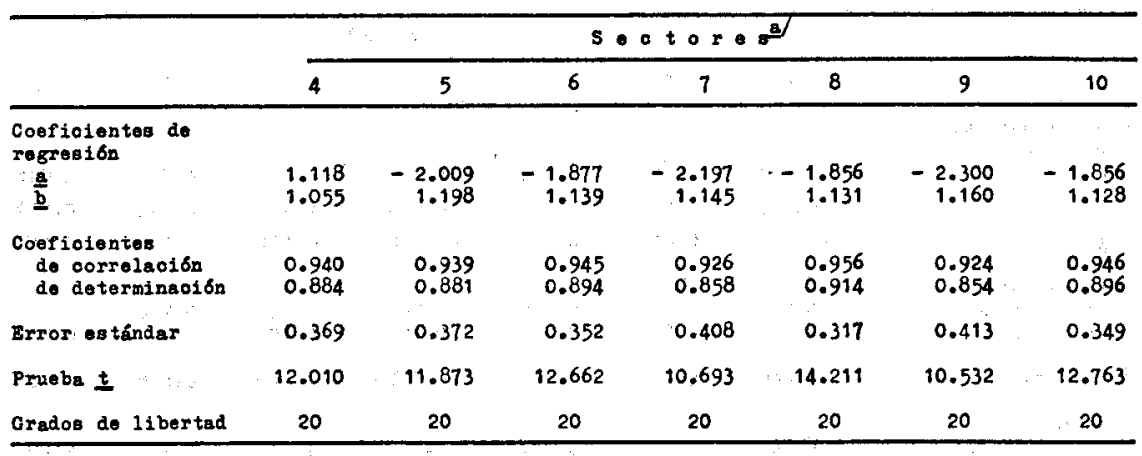

a Véase la nota del cuadro $6 \mathrm{~A}$.

Cuadro $8 \mathrm{~A}$

Resultados de LA FUNCión 3: $\ln (v / l)=a_{2}+b_{2} \ln (Y / N)$, CON LAS CIFRAS DE LOS PAÍSES DE LA MUESTRA, 1965

\begin{tabular}{|c|c|c|c|c|c|c|c|}
\hline \multirow[b]{2}{*}{$\therefore$} & \multicolumn{7}{|c|}{$s \cdot 0 t 0 r \in 8^{3}$} \\
\hline & 4 & 5 & 6 & 7 & 8 & 9 & 10 \\
\hline \multicolumn{8}{|l|}{$\begin{array}{l}\text { Coefioientes de } \\
\text { regresión }\end{array}$} \\
\hline$\underline{\mathrm{a}}$ & $\begin{array}{l}4.399 \\
0.601\end{array}$ & $\begin{array}{l}3.870 \\
0.614\end{array}$ & $\begin{array}{l}3.675 \\
0.687\end{array}$ & $\begin{array}{l}4.403 \\
0.671\end{array}$ & $\begin{array}{l}3.506 \\
0.733\end{array}$ & $\begin{array}{l}5.062 \\
0.530\end{array}$ & $\begin{array}{l}3.727 \\
0.682\end{array}$ \\
\hline $\begin{array}{l}\text { Coeficiente日 } \\
\text { de correlación } \\
\text { de determinaoion }\end{array}$ & $\begin{array}{l}0.832 \\
0.692\end{array}$ & $\begin{array}{l}0.900 \\
0.810\end{array}$ & $\begin{array}{l}0.910 \\
0.828\end{array}$ & $\begin{array}{l}0.847 \\
0.717\end{array}$ & $\begin{array}{l}0.886 \\
0.785\end{array}$ & $\begin{array}{l}0.765 \\
0.584\end{array}$ & $\begin{array}{l}0.904 \\
0.817\end{array}$ \\
\hline Brror eständar & 0.434 & 0.321 & 0.339 & 0.455 & 0.415 & 0.483 & 0.348 \\
\hline Prueba $\underline{t}$ & 6.530 & 8.997 & 9.550 & 6.941 & 8.328 & 5.163 & 9.236 \\
\hline Grados de libertad & 20 & 20 & 20 & 20 & 20 & 20 & 20 \\
\hline
\end{tabular}

a Véase la nota del cuadro $6 \mathrm{~A}$. 
Cuadro $9 \mathrm{~A}$

Resultados de la función 4: $\ln (v / l)=a_{3}+b_{3} \ln (Y / N)+c \ln N$, CON LAS CIFRAS DE LOS PAÍSES DE LA MUESTRA, 1965

\begin{tabular}{|c|c|c|c|c|c|c|c|c|}
\hline & & \multicolumn{7}{|c|}{$s \cdot \circ t \circ r^{a}$} \\
\hline & & 4 & 5 & 6 & 7 & 8 & 9 & 10 \\
\hline $\begin{array}{l}\text { Coeficientes de } \\
\text { regresibn }\end{array}$ & & & & & & & son & $\because$ \\
\hline $\mathrm{s}_{3}$ & & 4.988 & 3.550 & 3.594 & 4.430 & 4.476 & 4.998 & 3.041 \\
\hline$\underline{b}_{3}$ & & 0.587 & 0.622 & 0.689 & 0.669 & 0.709 & 0.513 & 0.699 \\
\hline 2 & 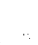 & -0.050 & 0.027 & 0.007 & -0.002 & -0.082 & 0.005 & 0.058 \\
\hline $\begin{array}{l}\text { Coeficientes } \\
\text { de oorrelaoibn } \\
\text { de determinacion }\end{array}$ & $\therefore$ & $\begin{array}{l}0.837 \\
0.700\end{array}$ & $\begin{array}{l}0.901 \\
0.813\end{array}$ & $\begin{array}{l}0.910 \\
0.828\end{array}$ & $\begin{array}{l}0.847 \\
0.717\end{array}$ & $\begin{array}{l}0.895 \\
0.802\end{array}$ & $\begin{array}{l}0.764 \\
0.584\end{array}$ & $\begin{array}{l}0.910 \\
0.828\end{array}$ \\
\hline Brror e日 tándar & & 0.440 & 0.328 & 0.348 & 0.467 & 0.409 & 0.496 & 0.348 \\
\hline $\begin{array}{c}\text { Prueba } t \\
\underline{b}_{3}\end{array}$ & & 6.140 & 8.724 & 9.107 & 6.591 & 7.979 & 4.923 & 9.255 \\
\hline 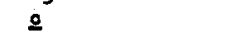 & & -0.698 & 0.509 & 0.121 & -0.031 & -1.236 & 0.067 & 1.028 \\
\hline Grados de libertad & & 20 & 20 & 20 & 20 & 20 & 20 & 20 \\
\hline
\end{tabular}

- Véase la nota del cuadro $6 \mathrm{~A}$.

\section{Cuadro $10 \mathrm{~A}$}

REsultados PARA El SECTOR MINERía Y EXPLOTACIÓN DE CANTERAS (2) CON LA Muestra de 16 PAÍses, 1963

\begin{tabular}{|c|c|c|c|}
\hline & \multicolumn{3}{|c|}{$F u n \circ 16 n$} \\
\hline & 1 & 2 & 3 \\
\hline \multicolumn{4}{|l|}{$\begin{array}{l}\text { Coeficientes de } \\
\text { regresibn }\end{array}$} \\
\hline$\underline{\mathbf{b}}$ & $\begin{array}{r}-0.104 \\
1.145\end{array}$ & $\begin{array}{l}5.698 \\
0.421\end{array}$ & $\begin{array}{l}2.115 \\
0.989\end{array}$ \\
\hline $\begin{array}{l}\text { Coofiolentes } \\
\text { de correleoibn } \\
\text { de determinaoion }\end{array}$ & $\begin{array}{l}0.967 \\
0.936\end{array}$ & $\begin{array}{l}0.516 \\
0.266\end{array}$ & $\begin{array}{l}0.932 \\
0.868\end{array}$ \\
\hline Exror estender & 0.308 & 0.704 & 0.443 \\
\hline Pruebe \pm & $14 \cdot 314$ & 2.255 & 9.591 \\
\hline Grados de libertad & 15 & 15 & 15 \\
\hline
\end{tabular}




\section{Cuadro $11 \mathrm{~A}$}

RESUltados para El SECTOR CONSTRUCCIÓN (11) CON LA MUESTRA DE 16 PAÍSES, 1965

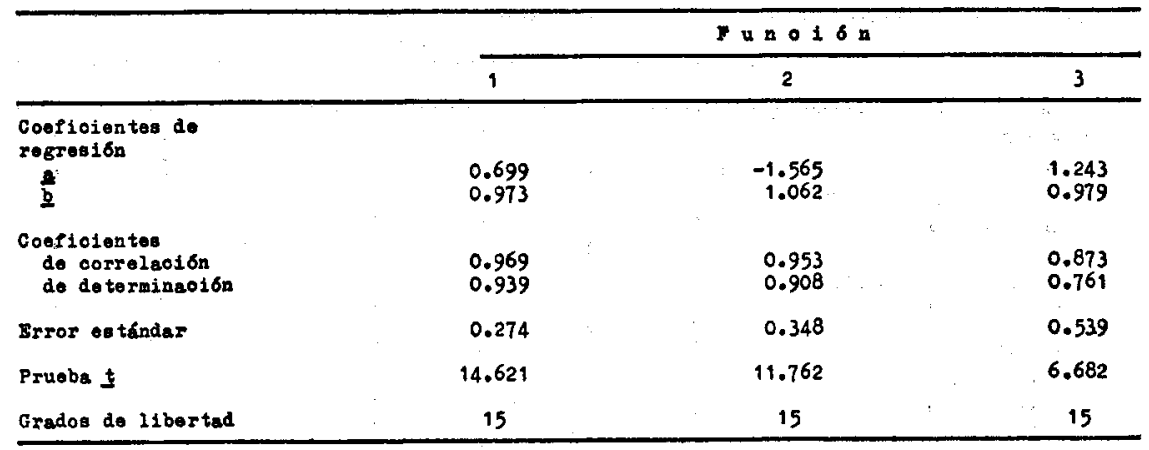

Cuadro $12 \mathrm{~A}$

MÉxico: Proyecciones del valor agregado y de la Fuerza DE TRABAJO PARA 1976

\begin{tabular}{|c|c|c|c|c|}
\hline \multirow[b]{2}{*}{ Sootor } & \multirow[b]{2}{*}{$\begin{array}{c}\text { Valor agregado } \\
\text { (1) }\end{array}$} & \multirow[b]{2}{*}{$\begin{array}{c}1 \\
(2)\end{array}$} & \multicolumn{2}{|c|}{$v / 1$} \\
\hline & & & $\begin{array}{c}\text { on pesos } \\
\text { (3) }\end{array}$ & $\begin{array}{c}\text { en d6larea } \\
\text { (4) }\end{array}$ \\
\hline 1 & 55036 & 10633 & 5176 & 414 \\
\hline 2 & 4395 & 153 & 28725 & 2298 \\
\hline 3 & 18782 & 100 & 187820 & 15026 \\
\hline 4 & 25269 & 994 & 25422 & 2034 \\
\hline 5 & 12668 & 436 & 29055 & 2324 \\
\hline 6 & 9413 & 167 & 56365 & 4509 \\
\hline 7 & 18467 & 302 & 61149 & 4892 \\
\hline 8 & 4748 & 126 & 37683 & 3015 \\
\hline 9 & 7784 & 142 & 54817 & 4385 \\
\hline 10 & 22904. & 1205 & 19007 & 1521 \\
\hline 11 & $21 \quad 148$ & 734 & 28812 & 2305 \\
\hline 12 & 11208 & 82 & 136683 & 10935 \\
\hline 13 & 121004 & 2192 & 55203 & 4416 \\
\hline 14 & 15264 & 463 & 32968 & 2637 \\
\hline 15 & 87086 & 1489 & 58486 & 4679 \\
\hline
\end{tabular}

Fuente: Columna 1: Departamento de Estudios Económicos, "Proyecciones de la economía mexicana para 1976", Banco de México, 1970; Columna 2: Departamento de Estudios Económicos, "Proyecciones de los principales aspectos de la economía mexicana", Banco de México, 1972.

a Millones de peos de 1960. 
Cuadro $13 \mathrm{~A}$

México: Productividad dL TRABajo Y TASAS de SALARIOS EN LA INDUSTRIA MANUFACTURERA, 1965

\begin{tabular}{|c|c|c|c|c|c|c|c|}
\hline & \multicolumn{7}{|c|}{ s.otor } \\
\hline & 4 & 5 & 6 & 7 & 8 & 9 & 10 \\
\hline Valor agregado bruto & 13443 & 7046 & 3892 & 5320 & 1782 & 2828 & 7626 \\
\hline Salarion & 4119 & 2853 & 1242 & 1501 & 581 & 1037 & 3243 \\
\hline $\begin{array}{l}\text { Nómero de trabajadoreab } \\
\text { Banco do Vexioo } \\
\text { Bstimaoion }\end{array}$ & $\begin{array}{l}617 \\
622\end{array}$ & $\begin{array}{l}431 \\
412\end{array}$ & $\begin{array}{l}158 \\
143\end{array}$ & $\begin{array}{l}155 \\
160\end{array}$ & $\begin{array}{l}75 \\
83\end{array}$ & $\begin{array}{l}96 \\
85\end{array}$ & $\begin{array}{l}484 \\
489\end{array}$ \\
\hline $\begin{array}{l}\text { Produotividad del trabajol } \\
\text { Banoo do Mbxioo } \\
\text { Batimaoion } \\
\text { Promedio }\end{array}$ & $\begin{array}{l}1744 \\
1728 \\
1736\end{array}$ & $\begin{array}{ll}1 & 309 \\
1 & 368 \\
1 & 338\end{array}$ & $\begin{array}{ll}1 & 972 \\
2 & 184 \\
2 & 078\end{array}$ & $\begin{array}{ll}2 & 748 \\
2 & 656 \\
2 & 702\end{array}$ & $\begin{array}{ll}1 & 902 \\
1712 \\
1807\end{array}$ & $\begin{array}{ll}2 & 359 \\
2 & 648 \\
2 & 503\end{array}$ & $\begin{array}{ll}1 & 261 \\
1 & 248 \\
1 & 254\end{array}$ \\
\hline $\begin{array}{l}\text { Pasa de malarios } \\
\text { Banoo de Mbxioo } \\
\text { Batimaoion } \\
\text { Promedio }\end{array}$ & $\begin{array}{l}535 \\
529 \\
532\end{array}$ & $\begin{array}{l}530 \\
554 \\
542\end{array}$ & $\begin{array}{l}629 \\
697 \\
663\end{array}$ & $\begin{array}{l}775 \\
749 \\
762\end{array}$ & $\begin{array}{l}620 \\
558 \\
584\end{array}$ & $\begin{array}{l}865 \\
971 \\
918\end{array}$ & $\therefore \begin{array}{l}536 \\
531 \\
534\end{array}$ \\
\hline
\end{tabular}

Fuente: Para el valor agregado, Banco de México, Cuentas nacionales y acervos de capital.

a Millones de pesos de 1960.

b En miles. Se incluye una cifra no oficial del Departamento de Estudios Económicos del Banco de México y una estimación derivada indirectamente proyectando la productividad del trabajo para 1965 con las cifras de 1960 y dividiendo la columna 1 por las cifras de la productividad del trabajo.

c En dólares. La productividad se obtiene dividiendo las cifras del valor agregado bruto por el número de trabajadores (con las cifras del Banco de México y las estimadas en este trabajo).

a En dólares. Las tasas de salarios se obtienen dividiendo las cifras de salarios entre el número de trabajadores (con los datos del Banco de México y los estimados en este trabajo). 
Cuadro $14 \mathrm{~A}$

MÉxico: Relación CAPITAL/TRABajo EN LA INDUSTRIA MANUFACTURERA, 1965

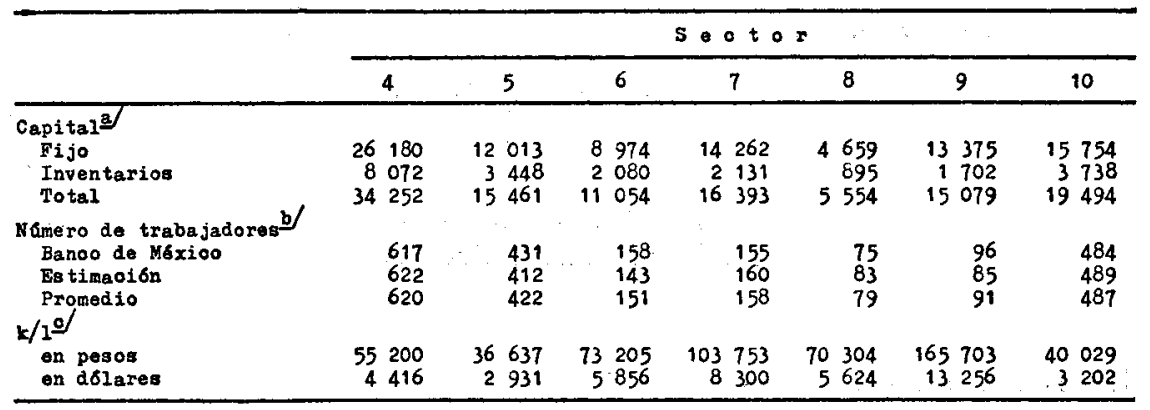

Fuente: Para las cifras de capital, Departamento de Estudios Económicos, Cuentas nacionales y acervos de capital, 1950-1967, Banco de México, 1969; para la fuerza de trabajo véase el cuadro $13 \mathrm{~A}$.

a Millones de pesos de 1960 .

b En miles. Se incluye una cifra no oficial del Banco de México; una estimación obtenida en este trabajo y el promedio de ambas.

c Capital total dividido por el número promedio de trabajadores. 